\title{
Formalizing the transformations of a cognitive universe
}

\author{
N. Lafaye de Micheaux ${ }^{1}$, G. Lopez ${ }^{1}$, P. Vitiello ${ }^{2}$ and J.L. Beauvois ${ }^{3}$ \\ ${ }^{1}$ Institut de Mathématiques de Luminy, CNRS UPR 9016, Marseille, France \\ ${ }^{2}$ Université des Sciences et Techniques de Saint-Jérôme, Marseille, France \\ ${ }^{3}$ Université de Sophia-Antipolis, Nice, France
}

\begin{abstract}
In an effort to continue the pioneering work of Harary in USA and Flament in France, we have undertaken to develop, on an experimental basis, a formalized theory of systems of beliefs and their modifications. This theory uses the psycho-social concepts of theories of cognitive consistency and of the tools of discrete mathematics, such as rewriting and intervals within graphs. The axioms and rewriting rules are elaborated from experimental data, and we demonstrate that the system we have built has the property of termination. This result is in accordance with experimental observations that show that every subject having an inconsistent system of beliefs (i.e., one containing contradictions) makes this system evolve towards consistency to reach a simple, consistent reference framework.
\end{abstract}

Keywords: rewriting, graphs of beliefs, consistency

\section{Introduction}

In the 1950's, social psychology could be seen endeavouring to theorize and formalize what was at the time called "cognitive universes", i.e., for a given individual, the universe of the elements of information or beliefs related to him(her)self, to objects, to other individuals and to the world as well as the relationships entertained among themselves by these information elements or beliefs ((Ba57), (Fes57), (Zaj68)).

The main idea of the theorists in the 50's was experimentally very fruitful ((Zaj68)) et has never been counter-argued ((DB96)): this idea is that cognitive universes tend towards a satisfactory "harmonious" state, said "state of consistency". Verbal definitions of such state of consistency vary according to diverse theories ((OT55), (Fes57), (AR58), (Hei44)). Although each of them has its specificity, even if not always self-evident, such theories are obviously not contradictory among themselves. Far from it, they all are grounded in a common basic formal representation, that of a "simple consistent reference framework" (or "consistent framework", (OT55)): The concepts of a cognitive universe are distributed among three disjoints sets, the set of concepts towards which the individual has a positive attitude, the set of concepts towards which the individual has a negative attitude, and the set of concepts not evaluated by the individual; between two evaluated concepts from the same set, there is a positive relation or no relation; between two evaluated concepts from different sets, a negative relation or no relation. This simple reference framework can be represented as a valued graph as in figure 1.

Theorists of cognitive consistency have in this way described certain features of cognitive universes: 


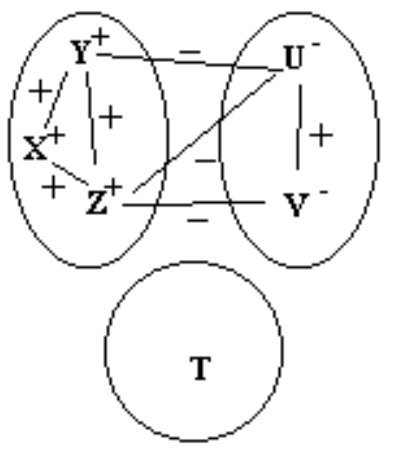

Fig. 1: A simple reference framework.

1- a tendency to approximate as much as possible a simple reference framework such as just defined (tendency towards consistency),

2- a tendency to establish the largest possible number of positive relations (tendency towards positiveness),

3- a tendency to establish relations where they do not exist (tendency towards completeness).

Such are the laws of the dynamics of any system of beliefs. It therefore becomes interesting to investigate how systems of beliefs may evolve when they lack initially this canonical simplicity.

\section{Structure of systems of beliefs}

The individual expresses beliefs regarding each pair of concepts and attitudes regarding each concept.

Let $S$ be an individual and $C$ the set of, at least, three concepts on which $S$ has to pronounce himself. Let us consider a pair $(x, y)$ of members of $C$, there are three possible beliefs:

1. $S$ believes they fit together,

2. $S$ believes they do not fit together,

3. $S$ does not know whether they fit together.

Axiom 1 For each pair $(x, y)$ of members of $C$ there exists one and only one belief.

This axiom allows us to define a structure of labeled graph in the following way:

Let $F$ be a set defined by $F=\{+,-, \lambda\}$ and let $G=\{C, C \times C, E\}$ be a graph such that $C$ is the finite set of concepts, $C \times C$ is the set of the edges and $E: C \times C \rightarrow F$ is a edge labeling function such that for each $(x, y) \in C \times C$,

- $E(x, y)=+$ if $x$ and $y$ fit together for $S$,

- $E(x, y)=-$ if $x$ and $y$ do not fit together for $S$,

- $E(x, y)=\lambda$ if $S$ does not know whether they fit together or not. 
Such a graph is called a graph of beliefs.

Definition 1 A graph of beliefs is said decision-complete if there is no edge labeled by $\lambda$.

If a cycle in a graph of beliefs has no edge labeled by $\lambda$, it is said closed for decision.

Axiom 2 For all $x \in C$ the individual $S$ has a unique attitude.

We can consider a new structure of graph describing the attitudes of $S$ and obtain a graph $G^{\prime}=$ $\{C, C \times C, E, A\}$ where $C$ and $E$ are defined as before and $A: C \rightarrow F$ is a vertex labeling function such that:

- $A(x)=+$ if $S$ has a positive attitude towards $x$,

- $A(x)=-$ if $S$ has a negative attitude towards $x$,

- $A(x)=\lambda$ if $S$ does not know whether he appreciates $x$ or not.

Such a graph is said a graph of beliefs with attitudes.

A labeled edge in such graphs $G$ or $G^{\prime}$ represents a belief, a labeled vertex in a graph of beliefs with attitudes $G^{\prime}$ represents an attitude in front of the concept associated to this vertex.

Definition 2 A graph of beliefs with attitudes is said decision-complete if there is no vertex labeled by $\lambda$.

\subsection{Consistency in graphs of beliefs}

Upset by the war, Lysistrata and Greek women induced their husbands to conclude peace. Lysistrata believed that women suffered too much from war, although men liked war, but at the same time women and men loved each other. We say that the relations between these three concepts are in an inconsistent state in the Lysistrata's system of beliefs. Therefore, she managed to change the relation between men and war to make this state consistent. We will obtain the simple reference framework in figure 2.

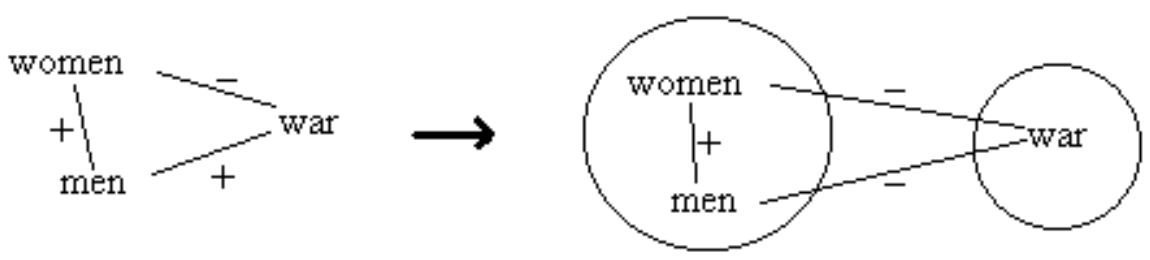

Fig. 2: Transformation of Lysistrata's graph of beliefs.

We observe that the product of the signs on the three relations has become positive in the second graph. In psycho-social theories we can find two definitions of consistency:

Definition 3 A system of beliefs is globally consistent if it can be represented by a graph $G$ which is a simple reference framework. If it is not globally consistent then it is said globally inconsistent. 
Definition 4 A system of beliefs is locally consistent if the graph $G$ representing the system has no cycle closed for decision where the product of the signs labeling the edges is negative. If not, then it is said locally inconsistent.

An elementary graph of beliefs has three vertices. If such a graph is decision-complete it is called a triad.

Definition 5 Let $G$ be a graph of beliefs and $k$ an integer. $G$ is $k$-consistent if $G$ has no cycle closed for decision, of length $k$, which is inconsistent.

Therefore a graph without an inconsistent triad is 3-consistent.

Proposition 6 A decision-complete graph of beliefs $G$ is 3-inconsistent if and only if there is $k \geq 3$ such that $G$ is $k$-inconsistent.

Proof: The direct proposition is obvious. By induction on $k$, it is easy to show that $k$-inconsistency implies 3-inconsistency: It is true for $k=3$. Assume it is true for $k=n$, and let $C_{n+1}=\left\{x_{1}, x_{2}, x_{3}, \ldots, x_{n+1}\right\}$ be an inconsistent cycle of length $n+1$. If the cycle $C_{n}=\left\{x_{1}, x_{3}, x_{4}, \ldots, x_{n+1}\right\}$ is inconsistent then $G$ is 3inconsistent by induction hypothesis. If it is consistent then the triad $\left\{x_{1}, x_{2}, x_{3}\right\}$ is inconsistent and $\mathrm{G}$ is 3-inconsistent. $\diamond$

The equivalence between the two definitions of consistency for decision-complete graphs is given by the following theorem:

Theorem 7 A decision-complete graph of beliefs is globally consistent if and only if it is locally consistent.

Proof: The direct proposition is obvious. To show that local consistency implies global consistency we observe that, in a decision-complete locally consistent graph $G$, if $A$ is a decision-complete sub-graph with edges labeled only by + and maximal for this property, then for all edges $(a, b)$ in $G$ such that $a \in A$ and $b \notin A,(a, b)$ is labeled by -. For all $x \notin A, x$ vertex of $G$ distinct from $b$, the edge $(b, x)$ is labeled by + because $G$ is locally consistent. Therefore $G$ is a globally consistent graph of beliefs. $\diamond$

\subsection{Consistency in a graph of beliefs with attitudes}

If an individual likes smoking but wants to be a good athlete, he has to solve a problem and probably will change his attitude towards cigarette or sport. He believes that they do not fit together. We say that, being in an inconsistent state, he will try to transform this state in order to make it consistent. We provide an illustration of this state in figure 3 .

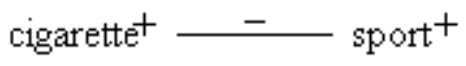

Fig. 3: Inconsistency in the smoking sportsmen's graph.

The bipartition of a decision-complete consistent graph of beliefs $G$ can be extended to a graph $G^{\prime}$ representing the same system of beliefs as $G$ but with attitudes. 
Definition 8 Let $G^{\prime}=\{C, C \times C, E, A\}$ be a graph of beliefs with attitudes. Then $G^{\prime}$ is said simply consistent if $G=\{C, C \times C, E\}$ is consistent.

If an individual likes smoking but wants to be a good athlete, he has to solve a problem and probably he will change his attitude in front of cigarette or sport. He knows that they do not fit together. We say that he is in an inconsistent state and he will try to transform this state and make it consistent.

The rules for A-local consistency, used by (AR58), can be expressed in our formalism by:

$$
\begin{aligned}
& A_{1}: \text { If } E(x, y)=+ \text { and } A(x)=+ \text { then } A(y)=+, \\
& A_{2}: \text { If } E(x, y)=+ \text { and } A(x)=- \text { then } A(y)=-, \\
& A_{3}: \text { If } E(x, y)=- \text { and } A(x)=+ \text { then } A(y)=-, \\
& A_{4}: \text { If } E(x, y)=- \text { and } A(x)=-\operatorname{then} A(y)=+.
\end{aligned}
$$

Definition 9 Let $G^{\prime}=\{C, C \times C, E, A\}$ be a graph of beliefs with attitudes, $G^{\prime}$ is said $A$-consistent if, for all $(x, y)$ in $C \times C$, the pair $(x, y)$ satisfies the conditions of the rules for $A$-local consistency.

Theorem 10 If $G^{\prime}$ is a decision-complete, simply inconsistent, graph of beliefs then it is A-inconsistent.

Proof: In an inconsistent cycle $C$ of $G^{\prime}$, the product of the signs labeling the edges must be negative. If $G^{\prime}$ was $A$-consistent, then we should have fulfilled an odd number of times the conditions $A_{3}$ and $A_{4}$ changing the sign of a vertex in $C$. As the cycle is closed the same vertex should have two distinct labels. This is impossible. $\diamond$

We will study first the properties of consistency or inconsistency in a decision-complete graph of beliefs and, using Proposition 6, we will only work locally on triads.

\section{Contiguity structure and transformations}

\subsection{Contiguity structure in a set of triads and classification}

The experiences made by the psycho-sociologists show that an individual has only a local perception of the relations between the concepts. Consequently the local approach is the best way to explore the transformations of a graph of beliefs.

Definition 11 In a graph of beliefs two triads $S$ and $T$ are contiguous if they have a common edge. Contiguity is said positive if the common edge has label $+($ denoted $S+T)$ and negative if the common edge has label $-($ denoted $S-T)$.

We can classify all the possible triads in a graph of beliefs:

1. $\mathbf{P}$ is the type of triads with only positive edges,

2. $\mathbf{M}$ is the type of triads with one positive and two negative edges,

3. $\mathbf{X}$ is the type of triads with only negative edges,

4. $\mathbf{Y}$ is the type of triads with two positive and one negative edges. 
The consistent triads are of type $\mathbf{P}$ or $\mathbf{M}$, the inconsistent ones are of type $\mathbf{X}$ or $\mathbf{Y}$.

In the experimental part of the study, psycho-sociologists have observed that, in a graph of beliefs, the individual, looking for more consistency, chooses to use a minimal number of transformations from his local point of view. He will try to eliminate first $\mathbf{X}$ and prefers to change a label - on an edge. We can represent these experimental data by metarules.

\subsection{Metarules}

An inconsistent graph will be affected by local transformations according to the following metarules:

1. Consistency metarule:

$\mathrm{M}_{1}$ : The transformations of the triads must eliminate inconsistency from a local point of view.

2. Contiguity metarule:

$\mathrm{M}_{2}$ : Two consecutive transformations are made, firstly, on contiguous triads.

3. Precedence Metarules:

$\mathrm{M}_{1}^{\prime}$ : The number of transformations must be minimal.

$\mathbf{M}_{2}^{\prime}$ : The elimination of the inconsistent triads gives priority to $\mathbf{X}$.

$\mathrm{M}_{3}$ : Priority is given to the transformation of negative contiguity.

The inconsistent triads of types $\mathbf{X}$ and $\mathbf{Y}$ will be locally considered, using $\mathbf{M}_{1}$, as variables in a rewriting system and those of types $\mathbf{M}$ and $\mathbf{P}$ as terminals.

The consistency metarule gives the following axiom:

Axiom 3 All triads in a system are consistent if and only if no transformation is possible in the system.

\subsection{Transformations and pyramids}

We have now to formalize the notion of transformation.

Definition 12 An elementary transformation $\tau$ consists in changing the label of an edge and is defined on the set of types of inconsistent triads by:

1. $\tau(\mathbf{X})=\mathbf{M}$,

2. $\tau(\mathbf{Y})=\mathbf{P}$ if the label - of an edge is changed in label + ,

3. $\tau(\mathbf{Y})=\mathbf{M}$ if the label + of an edge is changed in label - .

This notion can be extended to pairs of contiguous types of triads :

Definition 13 A binary transformation $\tau^{\prime}$ is defined on the set of pairs of types of contiguous inconsistent triads by:

For all contiguous pairs $(\mathbf{U}, \mathbf{V})$ of types of inconsistent triads, $\tau^{\prime}(\mathbf{U} \varepsilon \mathbf{V})=\tau(\mathbf{U}) \varepsilon^{\prime} \tau(\mathbf{V})$ where $\varepsilon$ is one of the labels + or - , and $\varepsilon^{\prime}=-\varepsilon$.

Then the precedence metarules imply: 
1. if two inconsistent triads are contiguous, then we have to transform their common edge instead of another one, eliminating two inconsistent triads instead of one,

2. the configurations $\mathbf{X}-\mathbf{X}$ are eliminated first, then the configurations $\mathbf{X}-\mathbf{Y}$,

3. then are eliminated the configurations $\mathbf{Y}-\mathbf{Y}$ and $\mathbf{Y}+\mathbf{Y}$ in this sequence.

In a decision-complete graph of beliefs $G$, a pair of contiguous triads forms a pyramid by completing the sub-graph created by this pair with the edge in $G$ between the two vertices which do not belong to the common edge.

All types of pyramids are:

1. Pyramids whose all faces are terminals,

(a) the pyramid $\mathbf{4 P}$ formed by four faces of type $\mathbf{P}$,

(b) the pyramid $\mathbf{4 M}$ formed by four faces of type $\mathbf{M}$,

(c) the pyramid $\mathbf{P 3 M}$ formed by one face of type $\mathbf{P}$ and three faces of type $\mathbf{M}$.

2. Pyramids with at least one face of type X. They are of type 4X, 2X2M, X3Y and XY2M, as shown in figure 4.

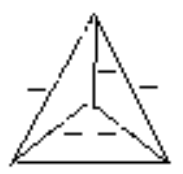

$\mathrm{X}$

$\mathrm{X}-\overline{\mathrm{X}}-\mathrm{X}$

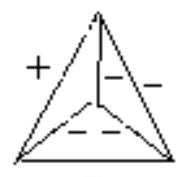

M

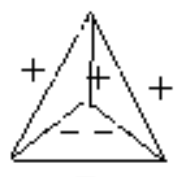

Y

$\mathrm{Y}-\overline{\mathrm{X}}-\mathrm{Y}$

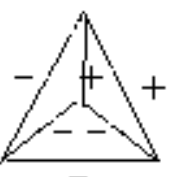

M

$\mathrm{M}-\overline{\mathrm{X}}-\mathrm{Y}$

Fig. 4: Pyramids with at least one face of type $\mathbf{X}$.

3. Pyramids with at least one face of type $\mathbf{Y}$ and no face of type $\mathbf{X}$. They are of type 2 Y2P, 4Y, 2Y2M and 2 YPM, as shown in figure 5.

\subsection{Rewriting rules}

In this description we can observe that each pyramid containing an inconsistent triad contains an even number of such triads. The binary transformation $\tau^{\prime}$ therefore can always be applied. The corresponding rewriting system is:

$$
\begin{aligned}
& \left(\mathbf{R}_{1}\right): \mathbf{X}-\mathbf{X} \longrightarrow \mathbf{M}+\mathbf{M}, \\
& \left(\mathbf{R}_{2}\right): \mathbf{X}-\mathbf{Y} \longrightarrow \mathbf{M}+\mathbf{P}, \\
& \left(\mathbf{R}_{3}\right): \mathbf{Y}-\mathbf{Y} \longrightarrow \mathbf{P}+\mathbf{P},
\end{aligned}
$$




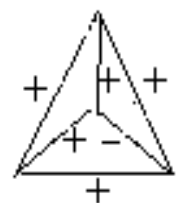

$\frac{\stackrel{P}{+}}{\mathrm{P}+\mathrm{V}}-\mathrm{Y}$
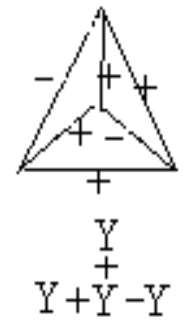
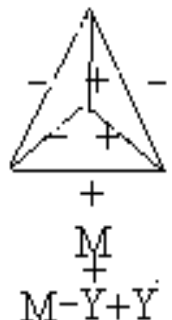
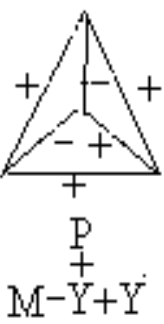

Fig. 5: Pyramids with at least one face of type $\mathbf{Y}$.

$\left(\mathbf{R}_{4}\right): \mathbf{Y}+\mathbf{Y} \longrightarrow \mathbf{M}-\mathbf{M}$.

The precedence rules give the sequence of applications of the rewriting rules:

The rule $\mathbf{R}_{1}$ is applied first, proceeding successively as often as possible through contiguous triads, to eliminate these occurrences. The rule $\mathbf{R}_{2}$ is applied when it is not possible anymore to apply $\mathbf{R}_{1}$ in the graph. Then the others rules are applied, in the same way, in the order of precedence, until it is not possible anymore to apply any rule.

\section{Propagation and termination for decision-complete graphs}

In all rewriting system the problem is the confluence and the termination of the system.

The problem of confluence in a graph rewriting system is given by the following question:

If it is possible to apply two rules in such a way that, having applied one, we cannot apply the other one, then, after applying a succession of possible rules, do we obtain the same graph whatever the choice made at the beginning ?

We do not need to solve this because it is irrelevant. Each individual establishing a unique succession gives its own preferences. He will try to construct his own consistent graph, which will not be the same as the graph of another individual having, at the beginning, the same graph of beliefs.

The only problem to solve is to know whether the individual will obtain, after a finite number of transformations, a consistent graph of beliefs. This would mean that the rewriting system has the property of termination.

Let $G$ be a decision-complete graph of beliefs. Applying one of the rewriting rules in a pyramid $\Pi$, with vertices $A, B, C, D$, in this graph, the pyramids concerned by a possible modification are among those constructed adding to the sub-graph $\Pi$ another vertex $S$ of $G$, distinct from $A, B, C$ and $D$, and the edges in $G$ joining $S$ to three vertices of $\Pi$. These pyramids are said concerned by the propagation. This is illustrated by figure 6 where the label modified by the application of the rule is evidenced by a circle.

The pyramids of vertex $S$ which will have an edge modified, are those whose common face with $\Pi$ is an inconsistent triad modified by the transformation. These pyramids, having one inconsistent face, will have at least two of it.

\subsection{Rules $\boldsymbol{R}_{1}, \boldsymbol{R}_{2}$ and $\boldsymbol{R}_{3}$}

In the pyramid $\Pi$, the application of a rule eliminates the two occurrences of inconsistent triads which are the common faces of each pyramid, concerned by the propagation, with $\Pi$ (in figure 6 the faces $A C D$ and 

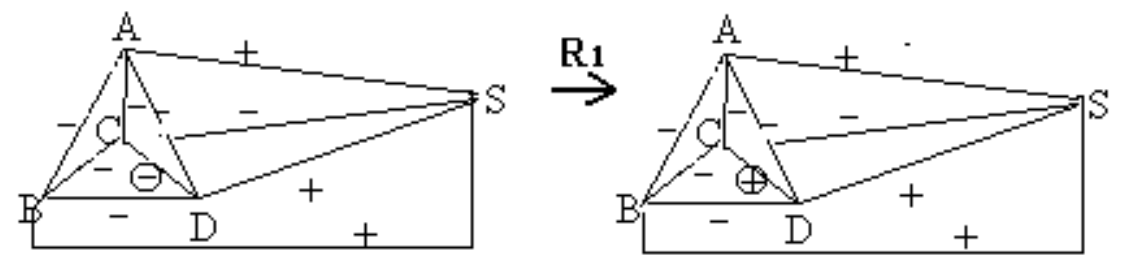

Fig. 6: Application of the rule $\mathbf{R}_{1}$.

$B C D)$. By applying the rule, the pyramid $\Pi$ will give a pyramid $\Pi^{\prime}$ with two inconsistent triads less and the two pyramids concerned by the propagation will give pyramids with one less occurrence of inconsistent triad, and, eventually, one more occurrence of inconsistent triad on their common face. This occurrence is said due to the propagation. The total number of occurrences of inconsistent triads is strictly diminished in such a sub-graph. This is true for each vertex $S$ in $G$ distinct of the vertices of $\Pi$.

Therefore we obtain the following property:

From a local point of view, the application of a rewriting rule strictly diminishes the number of inconsistent triads.

Definition 14 Two pyramids are adjacent if they have a common face.

Remark: The pyramid of type $\mathbf{4 P}$ can be adjacent with $\mathbf{P 3 M}$ which has a face of type $\mathbf{P}$ but not with 4M. But $\mathbf{4 M}$ can be adjacent with $\mathbf{4 M}$ and $\mathbf{P 3 M}$.

Definition 15 Two pyramids $\Pi_{1}$ and $\Pi_{2}$ are $i$-adjacent if they are adjacent and if their common face is an inconsistent triad. In this case we denote $\Pi_{1} /{ }^{i} \Pi_{2}$. Two pyramids $\Pi_{1}$ and $\Pi_{2}$ which are adjacent but not i-adjacent are said c-adjacent, denoted $\Pi_{1} /{ }^{c} \Pi_{2}$, their common face is a terminal triad.

According to the contiguity metarule, after applying a rewriting rule in a pyramid $\Pi$, the following application of the same rule is made in one of the two pyramids i-adjacent with $\Pi$ when it is possible. The application of one of the rules $\mathbf{R}_{1}, \mathbf{R}_{2}$ and $\mathbf{R}_{3}$ does not introduce a new inconsistent triad of type $\mathbf{X}$ because it changes a label + in a label -, but it can introduce an inconsistent triad of type $\mathbf{Y}$ by changing a terminal triad of type $\mathbf{M}$. The pair $\mathbf{X}-\mathbf{X}$ can be eliminated from the graph when it is not anymore possible to apply the rule $\mathbf{R}_{1}$ and cannot be introduced by applying $\mathbf{R}_{2}$ and $\mathbf{R}_{3}$. The pair $\mathbf{X}-\mathbf{Y}$ is eliminated from the graph when it is not anymore possible to apply the rule $\mathbf{R}_{2}$ and cannot be introduced by applying $\mathbf{R}_{3}$.

After the application of the rules $\mathbf{R}_{1}$ and $\mathbf{R}_{2}$ there is, in the graph, a finite number of triads of type $\mathbf{M}$ which can give a triad of type $\mathbf{Y}$ by propagation. The rule $\mathbf{R}_{3}$ will not introduce other triads of type $\mathbf{M}$ because $\mathbf{X}$ has been eliminated from the graph. Hence a chain of successive applications of this rule in the graph will end and then the pair $\mathbf{Y}-\mathbf{Y}$ will be eliminated.

The problem of the introduction of a new inconsistent triad of type $\mathrm{X}$ appears in the case of applying the rule $\mathbf{R}_{4}$ which transforms a label + in label - .

\subsection{The rule $\boldsymbol{R}_{4}$}

The rule is applied only on pyramids of type 2 Y2M and 2 YPM. 
At this stage, the rules $\mathbf{R}_{1}, \mathbf{R}_{2}$ and $\mathbf{R}_{3}$ have eliminated, in the graph, all the pyramids containing other pairs than the pair $\mathbf{Y}+\mathbf{Y}$, hence the only possible i-adjacent pyramids are of type 2Y2M or 2YPM.

Changing a label + in a label - , the propagation can introduce two types of inconsistent triads: The type $\mathbf{X}$ through transformation of a triad of type $\mathbf{M}$ containing the modified edge, or the type $\mathbf{Y}$ through transformation of a triad of type $\mathbf{P}$.

Due to these remarks, when we study all the possible combinations of i-adjacency, there remain only two cases:

1. The i-adjacency between $\mathbf{2} \mathbf{Y} \mathbf{2} \mathbf{M}$ and another pyramid when a triad of type $\mathbf{M}$ is modified by the propagation,

2. The i-adjacency between $\mathbf{2 Y P M}$ and $\mathbf{2 Y P M}$ when a triad of type $\mathbf{P}$ is modified by the propagation.

- In the first case, let $\Pi_{1}=A B C D$ be the first pyramid such that the faces $A C D$ and $B C D$ belong to type $\mathbf{Y}$ and the edge $C D$ has label + , and let $\Pi_{2}=S A C D$ be the second one. The edges $S A$ and $S B$ have the label + because $\mathbf{X}$ has been eliminated of the graph. It is an i-adjacency between to pyramids of type 2Y2M as in figure 7. We use the notion of interval in a graph allowing us to identify all the

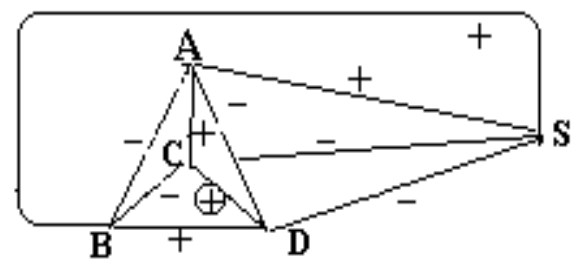

Fig. 7: An i-adjacency between to pyramids of type 2Y2M.

vertices in the graph in the same situation as $S$ and we observe that $G$ cannot have others vertices. Applying the rule $\mathbf{R}_{4}$, we obtain the sub-graph $\mathbf{4 M} /{ }^{c} \mathbf{X Y 2 M}$ which is not yet consistent. It is a new graph. We cannot apply the rule $\mathbf{R}_{1}$, but the rule $\mathbf{R}_{2}$, next in the sequence of applications of the rules, gives the sub-graph $\mathbf{4 M} /{ }^{c} \mathbf{P 3 M}$ which is consistent. Applying the rule $\mathbf{R}_{4}$ in the third pyramid, the graph becomes consistent.

The corresponding consistent reference framework is given in figure 8 .

- In the second case, let $\Pi_{1}=A B C D$ be the first pyramid such that the faces $A C D$ and $B C D$ belong to type $\mathbf{Y}$ and the edge $C D$ has label + , and let $\Pi_{2}=S A C D$ be the second one. The edge SB has label - , because $S A B D$ cannot be of type $2 \mathbf{Y} 2 \mathbf{P}$ as seen in figure 9. Each vertex of $G$ is, then, in situation of interval with one of the the vertices of this sub-graph. By applying the rule $\mathbf{R}_{4}$ first in $\Pi_{1}$ then in $\Pi_{2}$ we obtain the consistent reference framework given in figure 10 .

We obtain the theorem:

Theorem 16 A decision-complete graph of beliefs can be transformed into a consistent reference framework through a finite number of applications of the rewriting rules. 


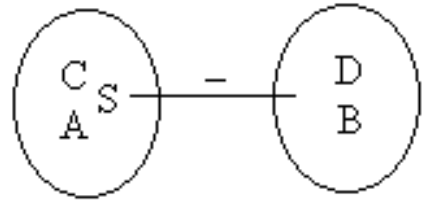

Fig. 8: The corresponding consistent reference framework.

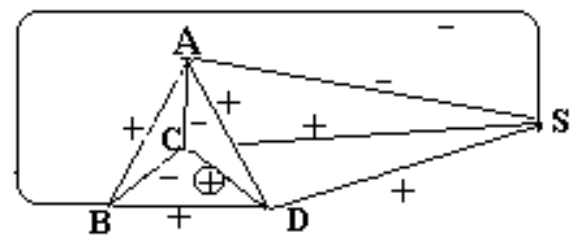

Fig. 9: An i-adjacency between to pyramids of type 2Y2P.

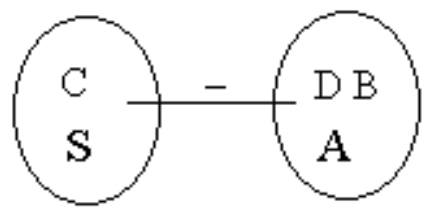

Fig. 10: The corresponding consistent reference framework. 


\section{Structures with attitudes}

The aim is now to obtain a similar result for graphs of beliefs with attitudes.

Definition 17 Let $G$ be the decision-complete graph of beliefs of an individual $J$ and $A$ the application defining the attitude of $J$ with relation to the concepts represented by $G$. Let $G_{A}$ be the graph constituted by $G$ and two new vertices $\beta$ and $\mu$ such that:

1. for each concept appreciated by $J$ there is an edge labeled + between the corresponding vertex and $\beta$ and an edge labeled - between the corresponding vertex and $\mu$,

2. for each concept not appreciated by $J$ there is an edge labeled - between the corresponding vertex and $\beta$ and an edge labeled + between the corresponding vertex and $\mu$,

3. the vertices $\beta$ and $\mu$ are related by an edge labeled - .

$G_{A}$ is called the enriched graph of $G$ associated to $A$.

Theorem 18 Let $G$ be a decision-complete graph of beliefs and A an application defining attitudes such that the graph with attitudes $G^{\prime}=(G, A)$ is decision-complete. $G^{\prime}$ is locally A-consistent if and only if the graph of beliefs $G_{A}$ is consistent.

Proof: We assume that $G^{\prime}$ is locally $A$-consistent. Therefore, by Theorem 10, $G$ is consistent. Let $S$ and and $T$ be vertices of $G_{A}$. The vertices $S, \beta$, and $\mu$ form a triad. Such a triad is consistent by construction. The vertices $S, T$, and $\beta$ (resp. $\mu$ ) form a consistent triad by $A$-consistency of $G^{\prime}$. Therefore $G_{A}$ is consistent. On the other side if $G_{A}$ is consistent then the vertices $\beta$ and $\mu$ cannot belong to a triad of type $\mathbf{X}$ or $\mathbf{Y}$, which means that the conditions for $A$-local consistency $A_{1}, A_{2}, A_{3}$ and $A_{4}$ are fulfilled in $G^{\prime} . \diamond$

Given $(G, A)$, a decision-complete graph of beliefs with attitudes, it is possible to construct $G_{A}$ the enriched graph of beliefs associated to $(G, A)$ and obtain an $A$-consistent graph by applying the rules of the rewriting system to $G_{A}$.

\section{Conclusion}

Graph theory has been used to formalize systems resorted to in social perception science (theory of equilibrium). In this research, in a first phase, we have developed a minimal formalism : graphs of beliefs. Two difficulties have then emerged in relation with, on one hand, the multiple graphs generated by one and the same individual, i.e. their transformations, on the other hand, the comparison of graphs generated by different individuals. In order to analyze the dynamics of graphs of beliefs, we have substituted them with systems of triads and obtained a rewriting system which has the property of termination, in accordance with the psycho-social theories of consistent cognitive universes. The second difficulty then boils down to comparing the dynamics of two different systems. The transformation chains, which we have brought to light, may allow an easier handling of this issue. 


\section{References}

[AR58] R.P. Abelson and M.J. Rosenberg. Symbolic psycho-logic: a model of attitudinal cognition. Behavioral Science, 3:1-13, 1958.

[Ba57] J. Bruner and al. Contemporary approaches to cognition. Harvard University Press, Cambridge, 1957.

[DB96] J.C. Deschamps and J.L. Beauvois. Des attitudes aux attributions: Sur la construction de la réalité sociale. Presses Universitaires de Grenoble, Grenoble, 1996.

[Fes57] L. Festinger. A Theory of cognitive dissonance. Stanford University Press, Stanford, 1957.

[Hei44] F. Heider. A Theory of cognitive dissonance. Wiley, New York, 1944.

[OT55] C.E. Osgood and P.H. Tannenbaum. The principe of congruity in the prediction of attitude change. Psychological Review, 62:42-55, 1955.

[Zaj68] R.K. Zajonc. Cognitive theories in social psychology, volume 1 of Handbook of Social Psychology. Addison-Wesley, Reading, 1968. 
